\title{
Food Safety During and After the Era of COVID-19 Pandemic
}

\author{
Amin N. Olaimat ${ }^{1 *}$, Hafiz M. Shahbaz ${ }^{2 *}$, Nayab Fatima ${ }^{2}$, Sadia Munir ${ }^{3}$ and \\ Richard A. Holley ${ }^{4}$ \\ 1 Department of Clinical Nutrition and Dietetics, Faculty of Applied Medical Sciences, The Hashemite University, Zarqa, \\ Jordan, ${ }^{2}$ Department of Food Science and Human Nutrition, University of Veterinary and Animal Sciences, Lahore, Pakistan, \\ ${ }^{3}$ College of Food Science and Technology, Huazhong Agricultural University, Wuhan, China, ${ }^{4}$ Department of Food \\ and Human Nutritional Sciences, University of Manitoba, Winnipeg, MB, Canada
}

\section{OPEN ACCESS}

Edited by:

Gian/uigi Mauriello,

Università degli Studi di Napoli

Federico II, Italy

Reviewed by:

Fohad Mabood Husain, King Saud University, Saudi Arabia

Liang (Leon) Wang,

Xuzhou Medical University, China

*Correspondence:

Amin N. Olaima

aminolaimat@hu.edu.jo;

amin_olaimat@yahoo.com

Hafiz M. Shahbaz

muhammad.shahbaz@uvas.edu.pk;

shahbaz.uaf@gmail.com

Specialty section:

This article was submitted to

Food Microbiology,

a section of the journal

Frontiers in Microbiology

Received: 15 May 2020

Accepted: 15 July 2020

Published: 04 August 2020

Citation:

Olaimat AN, Shahbaz HM, Fatima N, Munir S and Holley RA (2020) Food Safety During and After

the Era of COVID-19 Pandemic.

Front. Microbiol. 11:1854

doi: 10.3389/fmicb.2020.01854
The coronavirus disease 2019 (COVID-19) is a clinical syndrome caused by severe acute respiratory syndrome corona virus-2 (SARS-CoV-2). COVID-19 was declared a pandemic by the World Health Organization (WHO) on March 11, 2020 due to its rapid and extensive spread among many countries through its very contagious nature and its high mortality among the elderly and infirm. Recently, data on the survival of SARS-CoV2 on contact surfaces has been reported, but there is none on the survival of COVID-19 on food surfaces and packages. The potential survival and transmission of SARS-CoV-2 on/via food and packages are discussed based on data available for other respiratory viruses such as SARS-CoV and MERS-CoV. However, studies are needed to explore its transmission via food and survival on food packaging materials. The implementation of food safety management systems such as Hazard Analysis and Critical Control Points (HACCP), and Good Manufacturing Practices (GMP) are important to reduce the risk of COVID-19 infection. Cleaning, sanitation, good hygienic practices, and active packaging are also needed from farm to fork.

Keywords: coronavirus, COVID-19, SARS-CoV-2, food safety, food package, sanitation, transmission, active packaging

\section{INTRODUCTION}

In December 2019, SARS-CoV-2 was initially detected in patients who suffered from unusual viral pneumonia in Wuhan, Hubei, China (Kaul, 2020; Naserghandi et al., 2020; Petrosillo et al., 2020). The virus was first named 2019 novel coronavirus (2019-nCoV) by the WHO and later, when it was found that $86.9 \%$ of the novel virus genome was similar to the SARS-CoV genome, the virus was renamed SARS-CoV-2 (Chang et al., 2020; The Lancet Infectious Diseases, 2020). COVID-19 is the clinical syndrome caused by SARS-CoV-2 infection which is characterized by a respiratory disease with symptoms ranging from mild influenza (flu-like) to severe pneumonia and acute respiratory distress syndrome (Petrosillo et al., 2020). The clinical manifestations of COVID19 are non-specific and variable among patients, and between countries. Generally, COVID-19 symptoms involve fever, sore throat, runny or stuffy nose, dry cough, headache, myalgia or fatigue, sputum production, dyspnea, chest pain or pressure, joint pain, chills, loss of taste or smell, and a rash on the skin or discoloration of toes or fingers. Abdominal pain, dizziness, diarrhea, nausea, and vomiting are less common symptoms (Kaul, 2020; Naserghandi et al., 2020; Petrosillo et al., 2020). Borges do Nascimento et al. (2020) found that COVID-19-related symptoms among 59,254 patients in 61 studies were: fever (82\%), cough (61\%), muscle aches and/or fatigue (36\%), dyspnea $(26 \%)$, headache $(12 \%)$, sore throat (10\%), and gastrointestinal symptoms (9\%). On average, the 
incubation period takes 5-6 days for a patient to show the symptoms after infection, however, it may reach up to 14 days (World Health Organization [WHO], 2020).

COVID-19 infection is highly contagious among the population and now almost all countries have reported cases and deaths. On March 11, 2020, COVID19 was characterized as a pandemic by the WHO. As of early July, over 12 million confirmed cases and 550,000 deaths of COVID-19 have been reported worldwide (World Health Organization [WHO], 2020).

\section{COVID-19 TRANSMISSION}

At the beginning of the pandemic, two-third or 27 out of 41 reported cases had previously visited the Huanan seafood wholesale market where live animals were sold close to seafood and meat products, suggesting that the virus was transmitted from animals to humans (Guan et al., 2020; Harapan et al., 2020; Naserghandi et al., 2020). The genome of SARS-CoV-2 is closely related to the genomes of the SARS-CoV that caused the SARS epidemic during 2003 and the SARS-related-CoVs (SARSr$\mathrm{CoVs}$ ) that was isolated from horseshoe bats. This suggested that the primary host of SARS-CoV-2 was a bat and other animals including pigs, or pangolins are potential secondary hosts to the virus (Lai et al., 2020; Sun et al., 2020).

To date, the impact of the seafood market in spreading COVID-19 is not fully understood (Harapan et al., 2020). It has been proposed that SARS-CoV-2 was introduced to the seafood market in Wuhan, Hubei, China and then the disease spread more rapidly through human-to-human interactions, which has been confirmed by the occurrence of infection among the family members and medical workers attending the victims (Chan et al., 2020; Yu et al., 2020). The most plausible transmission routes are respiratory droplets dispersed via talking, sneezing and coughing or direct contact with infected persons. Other suggested routes are fecal-oral transmission, contaminated fomite transmission and mother-fetal vertical transmission (Naserghandi et al., 2020; Wang et al., 2020).

It has been demonstrated that COVID-19 can also be transmitted by asymptomatic patients who are harboring the virus during its early incubation period before symptoms appear (Li et al., 2020; Ye et al., 2020; Zhang et al., 2020). Additionally, SARS-CoV-2 has reappeared in recovered patients leading to a reoccurrence of illness. This has been confirmed through the detection of viral nucleic acid using a real-time RT-PCR after patient recovery and discharge from the hospital (Chen et al., 2020; Lan et al., 2020; Qiao et al., 2020). However, the exact reason for the reappearance of SARS-CoV-2 is not well understood.

\section{DISCUSSION}

\section{The Potential COVID-19 Transmission via Food Products}

Foodservice operators were among the first workers in frontline employment sectors experiencing the impact of the COVID-19 pandemic. However, there is no study to date which reports that COVID-19 spreads via food products. Further, no evidence is available showing that viruses which infect the respiratory tract can be transmitted via food or food packaging (Food and Agriculture Organization of the United Nations [FAO] and World Health Organization [WHO], 2020). The transmission of SARS-CoV and MERS-CoV through the consumption of foods does not appear to have occurred yet (European Food Safety Authority [EFSA], 2020). However, it has been reported that human coronavirus 229E (HuCoV-229E) survived for at least 5 days on the surfaces of polyvinyl chloride (PVC), polyfluorotetraethylene (Teflon, PTFE), glass, ceramic tiles, and stainless steel and for 3 days on silicon rubber surfaces at $21^{\circ} \mathrm{C}$ with a relative humidity of $30-40 \%$ (Warnes et al., 2015). Similarly, SARS-CoV-2 survived on stainless steel and plastic up to 2 and 3 days, respectively, at 21$23^{\circ} \mathrm{C}$ and a relative humidity of $40 \%$; however, the virus was not detected on copper and cardboard, after 4 and $24 \mathrm{~h}$, respectively (van Doremalen et al., 2020). These results indicated that SARS-CoV-2 can be transmitted via contact surfaces because of the ability of the virus to survive on the surfaces for several days.

Coronaviruses can persist for long periods in environmental samples which may enhance the probability of transmission via package contact surfaces (Geller et al., 2012). It has been confirmed that virulence of variola (smallpox) virus and influenza virus were positively correlated with survival time in the external environment, which explained their high mortality rate compared to other viruses with low survival rates in environmental samples such as the virus causing parainfluenza and rhinovirus (Walther and Ewald, 2004).

Food and Agriculture Organization of the United Nations [FAO] and World Health Organization [WHO] (2020) proposed that touching food packages or containers contaminated with SARS-CoV-2 could transmit the virus to the mouth, nose, or eyes. However, this is not considered the main route for disease spread because the virus shows poor survival on these surfaces. A previous study reported that food products were a plausible transmission route for respiratory viruses including SARS-CoV-1 and influenza (Klein, 2004). In another study, the risk of Ebola infection to individual humans in the United States resulting from contaminated cocoa beans, palm oil, or cashews imported from South Africa was considered negligible to low (Bergeron et al., 2016). In addition, several studies showed that transmission of avian influenza through poultry products (Golden et al., 2009; Bauer et al., 2010; Sánchez-Vizcaíno et al., 2010) or water consumption was a remote possibility, but possible (Schijven et al., 2005). Similarly, the probability that consumers from the United Kingdom might get infected with COVID-19 via the consumption of food or the handling of material contacting food or packaging was considered negligible to very low. As mentioned, the genome of SARS-CoV-2 is closely related to SARS-CoV for which the transmission via foods has not been confirmed. It has been suggested that the potential foodborne transmission of SARS-CoV-2 may occur due to the consumption of foods originating from infected 
animals or the consumption of cross-contaminated foods (Oakenfull and Wilson, 2020).

More effort is needed to address the transmission of SARSCoV-2 from the respiratory tract to food package surfaces or through food consumption. The Food and Drug Administration [FDA] (2020b) proposed guidelines for consumers during food shopping, food handling and food preparation. It is worth mentioning that food handlers including food establishment employees and consumers should adhere to good sanitation and hygienic practice guidelines to avoid SARS-CoV-2 transmission and comply with at least the minimum requirements of the food safety system.

\section{The Potential Survival of SARS-COV-2 in Food Products}

It is widely known that viruses cannot multiply in food products because they need an animal or human host to grow. However, to date, no study has investigated the survival of SARS-CoV2 in foods. To the best of our knowledge, only two studies reported the survival of infectious respiratory viruses in food products. Adenovirus survived on both lettuce and strawberries at $4^{\circ} \mathrm{C}$ for up to 10 days. In contrast, coronavirus survived only 2 days on lettuce, and it was not recovered from the surface of strawberries after inoculation (Yépiz-Gómez et al., 2013). These results indicated that respiratory viruses may transfer from food surfaces to the hands and subsequently to the mouth, nose or eyes. The survival of MERS-CoV in different types of milk (camel, goat and cow milk) at 4 or $22^{\circ} \mathrm{C}$ has been investigated. MERS-CoV titers were decreased by less than $1 \log$ in all types of milk after $72 \mathrm{~h}$ at $4^{\circ} \mathrm{C}$. Higher $\log$ reductions were observed when milk was stored at $22^{\circ} \mathrm{C}$ since the virus titers decreased by $\leq 2.0 \log$ with $48 \mathrm{~h}$ of storage. Low temperature, long time pasteurization $\left(63^{\circ} \mathrm{C} / 30 \mathrm{~min}\right)$ of raw milk completely eliminated the virus from the milk of the three different animals (van Doremalen et al., 2014).

The infectious dose of most respiratory viruses is low; thus, the handling or consumption of food products could represent a risk for infection. As a result, preventive measures such as washing and sanitizing of fresh produce surfaces as well as the implementation of good personnel hygiene and practices among workers would seem reasonable ways to reduce the risk of virus transmission.

Another issue is that several viruses that cause respiratory infections have been found in the human gastrointestinal tract and were capable of proliferation there. These include Enterovirus (Coxsackie A, B virus), Parechovirus, Orthomyxovirus (Avian influenza virus), Henipavirus (Nipahand Hendra viruses), Mastadenovirus (adenovirus), Alphatorquevirus (Torque Teno virus), and coronavirus (Bosch et al., 2018). In the later case, lymphocytes and mucosal epithelial cells of the patients' intestine were positive for SARS-CoV (Shi et al., 2005). This may mean that the viruses can be acquired by humans via the consumption of contaminated foods. However, these results may not apply to SARS-CoV-2, which points out the need for studies to investigate the survival of SARS-CoV-2 in different foods and on food packages.

\section{Need for Food Hygiene Practices From Farm to Fork}

As mentioned, COVID-19 virus has an ability to stay alive for up to $72 \mathrm{~h}$ as a virion on inanimate objects after completing its life cycle in the body of an infected person (van Doremalen et al., 2020). Therefore, if the respiratory discharges of the COVID-19 patient come in contact with food, the food items can become a fomite (carrier), and if these items are contacted by other individuals, the virus is more likely to gain entry to their respiratory epithelium when unsanitized hands touch the nose, eyes, and mouth (Bundesinstitut für Risikobewertung [BfR], 2020; Centers for Disease Control and Prevention [CDC], 2020).

The surfaces of utensils, packaging material, counters, conveyor belts, interiors of transport vehicles, and all other food work stations where there might be human contact with food should remain a focus of attention where food handlers can act to impede the spread of COVID-19. Therefore, the proper use of personal protective equipment and adherence to the guidelines issued by public health authorities that include regular hand washing when exchanging goods, plus the use of hand sanitizers, wearing masks and gloves, and the maintenance of at least 6 feet between personnel are most important. A range of disinfectants and sanitizers are available in the marketplace. If disinfectant labels suggest that they are effective against coronaviruses or norovirus, then they should be effective against SARS-CoV-2 as well. Additionally, complete instructions are given on EPA disinfectant labels regarding the contact time, concentration, and appropriate surfaces for application (Environmental Protection Agency, 2020).

\section{Food Industries}

All food industry organizations should strictly follow the protocols of Food Safety Management Systems (FSMS) given by authorities based on HACCP principles and should be kept updated in response to new pieces of evidence for viruses when required. In food companies where HACCP protocols are not being implemented, an expert should be appointed who will remain in contact with public health authorities to seek advice during the pandemic situation. Hand washing stations should be maintained for the workforce with the provision of normal soap, warm running water, hand sanitizers, and posters designed for displaying information regarding effective hand washing and sanitization. The physical distancing of 6 feet should be implemented among workers as infected people may remain asymptomatic or be pre-symptomatic during the course of the disease and may spread the infection when close to others (Kimball et al., 2020; Pan et al., 2020; Tong et al., 2020; Wei et al., 2020; Yu et al., 2020). The introduction of staggered workstations is an effective method to overcome the challenge of physical distancing in food industry facilities.

\section{Food Delivery}

It is advised to minimize the contact between people during the outbreak; therefore, online food deliveries are more desirable. These allow physical distancing between customers and sales 
personnel. At this stage, proper dissemination of information on food handling practices is also required. Since food packages and paper currency are exchanged between consumers and retailers, proper precautions are needed to minimize the potential for virus transfer during the transaction. Some third-party delivery companies have also introduced contact-free delivery to homes. The packaging can be discarded after keeping track of important information mentioned on it. The proper use of gloves, sanitizers, and disinfectants can minimize the risk of virus spread and disease transmission (Food and Agriculture Organization of the United Nations [FAO] and World Health Organization [WHO], 2020; Food and Drug Administration [FDA], 2020a).

\section{Retail Food Premises}

Maintaining the movement of food along the food chain is an important function that requires all involved to contribute and stay vigilant. It is necessary to maintain the confidence and trust of consumers regarding food safety and food availability. In case of limitations on the foodservice industry, home deliveries can be promoted, however, a safe and secure environment for food retail shops and canteens must be ensured at both the consumer's and retailer's end. Retailers can play their roles by ensuring the provision of sanitary facilities including wipes, disinfectants, sanitizers, and display of sanitary practices through visual aids. Physical distancing can be maintained by having the floors marked as a reference for maintaining minimum distance required. Plexiglass can be installed to avoid contact at cashier counters, and food tasting for promotional campaigns should be avoided (Food and Agriculture Organization of the United Nations [FAO] and World Health Organization [WHO], 2020; Food and Drug Administration [FDA], 2020a).

At the consumer end, people should make sure that family members belonging to vulnerable groups (immunecompromised, elderly, children, and COVID-19 patients) should stay at home (Centers for Disease Control and Prevention $[C D C], 2020)$. Besides, the use of masks, wearing gloves, using hand sanitizers, using wipes before handling food carts, avoiding reusable shopping bags and opting for acceptable respiratory etiquette should be prioritized. If reusable bags are deemed regionally acceptable, their disinfection should be done instantly after use.

\section{Home Kitchens}

It is advised to stock food items according to their perishability in order to minimize the number of visits to markets. After safe purchasing with all protocols, the most neglected thing is the safe handling of food. With respect to food handling practices in our kitchens, evidence that has been obtained with coronaviruses indicated that they cannot survive cooking, but if food remains unwashed and is frozen afterward, the virus can survive up to 2 years during frozen storage (Bundesinstitut für Risikobewertung [BfR], 2020). Therefore, scrubbing of food items like fruits and vegetables is essential if they are not to be cooked for longer periods or to be eaten uncooked. For canned foods, the lids should be wiped before opening. Ensure the disinfection of utensils, pots, countertops, and fridge at every use and minimize the risks of cross-contamination among food items during storage. In addition, respect cooking protocols to ascertain food safety and avoid a false sense of security by following proper cooking time, temperature, and thawing protocols.

\section{Need for Active and Intelligent Packaging}

Consumers' concern regarding the ability of SARS-CoV-2 to survive on the surface of packages has led to an increasing interest in the development of polymers and biopolymers with antiviral properties. The applications of polymers and biopolymers have shown high efficacy against hepatitis A virus (HAV) and human norovirus (HuNoV) (Randazzo et al., 2018). A previous study showed that the release of copper ions can help in the inactivation of HuCoV-229E on copper or copper alloy surfaces (Warnes et al., 2015). These findings are a source of validation for the recent findings by van Doremalen et al. (2020) regarding the decreased viability of SARS-CoV-2 on copper surfaces and inactivation within $2 \mathrm{~h}$. The lack of trials on food matrices and related food regulatory requirements remain the hurdles to the adoption of novel food packaging. The development of biopolymers with antiviral properties and their applications in the food area remains an open field of research. For example, it has been recently reported that the use of nanomaterial coatings or films containing copper, silver, and zinc nanoparticles has a potential against SARS-CoV-2 to prevent contamination of food packaging surfaces and thus reduce its transmission (Sportelli et al., 2020).

In conclusion, there are only shreds of evidence regarding the duration of coronavirus survival on different contact surfaces and in foods under certain conditions which suggests the need for advanced studies in understanding the risk of COVID-19 spread associated with food and food packages. Research trials are required to find a link between the ingestion of food contaminated with SARS-CoV-2 and the probability of infections as well as the development of antiviral active packaging using nano-based biopolymer materials. The current guidelines issued by public health authorities are based on the disease patterns of previously encountered coronaviruses and they need to be updated according to the novel coronavirus SARS-CoV-2 as this virus is likely to persist and people will have to modify their "normal behavior" to a "new normal."

\section{DATA AVAILABILITY STATEMENT}

All datasets generated for this study are included in the article/supplementary material.

\section{AUTHOR CONTRIBUTIONS}

AO, HS, and NF wrote the draft of the manuscript. AO, HS, NF, and SM reviewed and edited the final manuscript version. $\mathrm{RH}$ critically revised the final manuscript version. All authors contributed to the article and approved the submitted version. 


\section{REFERENCES}

Bauer, N., Dearfield, K., Dennis, S., Disney, W. T., Ebel, E., Evans, P., et al. (2010). Interagency Risk Assessment For The Public Health Impact Of Highly Pathogenic Avian Influenza Virus In Poultry, Shell eggs, and egg products. Food safety and inspection service (FSIS), Food and Drug Administration (FDA) and Animal and Plant Health Inspection Service (APHIS) Joint Document. Available online at: http://www.fsis.usda.gov/wps/wcm/connect/955e7a9a-24f8-4b31-982656485c06eab7/HPAI_Risk_Assess_May2010.pdf?MOD=AJPERES (accessed May 2, 2020).

Bergeron, J. G., Mann, E. M., Farnham, M. W., Kennedy, S., Everstine, K., Prasarnphanich, O. O., et al. (2016). Rapid-response risk evaluation of Ebola spread via the food system. IBM J. Res. Dev. 60:778. doi: 10.1147/JRD.2016. 2585778

Borges do Nascimento, I. J., Cacic, N., Abdulazeem, H. M., von Groote, T. C., Jayarajah, U., Weerasekara, I., et al. (2020). Novel coronavirus infection (covid19) in humans: a scoping review and meta-analysis. J. Clin. Med. 9:941. doi: $10.3390 / \mathrm{jcm} 9040941$

Bosch, A., Gkogka, E., Le Guyader, F. S., Loisy-Hamon, F., Lee, A., van Lieshout, L., et al. (2018). Foodborne viruses: detection, risk assessment, and control options in food processing. Int. J. Food Microbiol. 28, 110-128. doi: 10.1016/ j.ijfoodmicro.2018.06.001

Bundesinstitut für Risikobewertung [BfR] (2020). Can The New Type Of Coronavirus Be Transmitted Via Food And Objects?. Available online at: https://www.bfr.bund.de/en/can_the_new_type_of_coronavirus_be_ transmitted_via_food_and_objects_-244090.html (accessed April 29, 2020).

Centers for Disease Control and Prevention [CDC] (2020). How Coronavirus Spreads. Atlanta: CDC.

Chan, J. F. W., Yuan, S., Kok, K. H., To, K. K. W., Chu, H., Yang, J., et al. (2020). A familial cluster of pneumonia associated with the 2019 novel coronavirus indicating person-to-person transmission: a study of a family cluster. Lancet 395, 514-523. doi: 10.1016/s0140-6736(20)30154-9

Chang, L., Yan, Y., and Wang, L. (2020). Coronavirus disease 2019: coronaviruses and blood safety. Transfus. Med. Rev. 34, 75-80. doi: 10.1016/j.tmrv.2020. 02.003

Chen, D., Xu, W., Lei, Z., Huang, Z., Liu, J., Gao, Z., et al. (2020). Recurrence of positive SARS-CoV-2 RNA in COVID-19: a case report. Int. J. Infect. Dis. 93, 297-299. doi: 10.1016/j.ijid.2020.03.003

Environmental Protection Agency (2020). List N: Disinfectants For Use Against SARS-CoV-2. Washington, DC: Environmental Protection Agency.

European Food Safety Authority [EFSA] (2020). Coronavirus: No Evidence That Food Is A Source Or Transmission Route. Parma: EFSA.

Food and Agriculture Organization of the United Nations [FAO] and World Health Organization [WHO] (2020). COVID-19, And Food Safety. Guidance For Food Businesses. Interim Guidance, April, 1-6. Geneva: WHO.

Food and Drug Administration [FDA] (2020a). Food Safety and the Coronavirus Disease 2019 (COVID-19). White Oak Campus: FDA.

Food and Drug Administration [FDA] (2020b). Shopping For Food During the COVID-19 Pandemic - Information For Consumers. April, 2020. White Oak Campus: FDA.

Geller, C., Varbanov, M., and Duval, R. E. (2012). Human coronaviruses: insights into environmental resistance and its influence on the development of new antiseptic strategies. Viruses 4, 3044-3068. doi: 10.3390/v4113044

Golden, N. J., Schlosser, W. D., and Ebel, E. D. (2009). Risk assessment to estimate the probability of a chicken flock infected with H5N1 highly pathogenic avian influenza virus reaching slaughter undetected. Foodborne Pathog. Dis. 6, 827-835. doi: 10.1089/fpd.2008.0253

Guan, W., Ni, Z., Hu, Y., Liang, W., Ou, C., He, J., et al. (2020). Clinical characteristics of coronavirus disease 2019 in China. N. Engl. J. Med. 382, $1708-1720$.

Harapan, H., Itoh, N., Yufika, A., Winardi, W., Keam, S., Te, H., et al. (2020). Coronavirus disease 2019 (COVID-19): a literature review. J. Infect. Public Health 13, 667-673.

Kaul, D. (2020). An overview of coronaviruses including the SARS-2 coronavirus Molecular biology, epidemiology and clinical implications. Cur. Med. Res. Pract. 10, 54-64. doi: 10.1016/j.cmrp.2020.04.001

Kimball, A., Hatfield, K. M., Arons, M., James, A., Taylor, J., Spicer, K., et al. (2020). Asymptomatic and presymptomatic SARS-COV-2 infections in residents of a long-term care skilled nursing facility - King County, Washington, March 2020. Morb. Mortal. Wkly Rep. 69, 377-381. doi: 10.15585/mmwr.mm6913e1
Klein, G. (2004). Spread of viruses through the food chain. Deut. Tierarztl. Woch. $111,312-314$.

Lai, C. C., Shih, T. P., Ko, W. C., Tang, H. J., and Hsueh, P. R. (2020). Severe acute respiratory syndrome coronavirus 2 (SARS-CoV-2) and coronavirus disease2019 (COVID-19): the epidemic and the challenges. Int. J. Antimicrob. Agents 55:105924. doi: 10.1016/j.ijantimicag.2020.105924

Lan, L., Xu, D., Ye, G., Xia, C., Wang, S., Li, Y., et al. (2020). Positive RT-PCR test results in patients recovered from COVID-19. JAMA 323, 1502-1503.

Li, Y., Shi, J., Xia, J., Duan, J., Chen, L., Yu, X., et al. (2020). Asymptomatic and symptomatic patients with non-severe coronavirus disease (COVID-19) have similar clinical features and virological courses: a retrospective single center study. Front. Microbiol. 11:1570. doi: 10.3389/fmicb.2020.01570

Naserghandi, A., Allameh, S. F., and Saffarpour, R. (2020). All about COVID-19 in brief. New Microb. New Infect. 35:100678. doi: 10.1016/j.nmni.2020.100678

Oakenfull, R. J., and Wilson, A. J. (2020). Qualitative Risk Assessment: What is The Risk Of Food Or Food Contact Materials Being A Source Or Transmission Route of SARS-CoV-2 for UK Consumers?. London: Food Standards Agency.

Pan, X., Chen, D., Xia, Y., Wu, X., Li, T., Ou, X., et al. (2020). Asymptomatic cases in a family cluster with SARS-CoV-2 infection. Lancet Infect. Dis. 20, 410-411. doi: 10.1016/s1473-3099(20)30114-6

Petrosillo, N., Viceconte, G., Ergonul, O., Ippolito, G., and Petersen, E. (2020). COVID-19, SARS and MERS: are they closely related? Clin. Microbiol. Infect. 26, 729-734. doi: 10.1016/j.cmi.2020.03.026

Qiao, X.-M., Xu, X.-F., Zi, H., Liu, G.-X., Li, B.-H., Du, X., et al. (2020). Re-positive cases of nucleic acid tests in discharged patients with COVID-19: a follow-up study. Front. Med. 7:349. doi: 10.3389/fmed.2020.00349

Randazzo, W., Fabra, M. J., Falcó, I., López-Rubio, A., and Sánchez, G. (2018). Polymers and biopolymers with antiviral activity: potential applications for improving food safety. Compr. Rev. Food Sci. 17, 754-768. doi: 10.1111/15414337.12349

Sánchez-Vizcaíno, F., Perez, A., Lainez, M., and Sánchez-Vizcaíno, J. M. (2010). A quantitative assessment of the risk for highly pathogenic avian influenza introduction into Spain via legal trade of live poultry. Risk Analy. 30, 798-807. doi: 10.1111/j.1539-6924.2009.01351.x

Schijven, J., Teunis, P., and de Roda Husman, A. (2005). Quantitative Risk Assessment Of Avian Influenza Virus Infection Via Water. RIVM Report 703719012/2005. Available online at: https://www.rivm.nl/bibliotheek/ rapporten/703719012.pdf (accessed May 5, 2020).

Shi, X., Gong, E., Gao, D., Zhang, B., Zheng, J., Gao, Z., et al. (2005). Severe acute respiratory syndrome associated coronavirus is detected in intestinal tissues of fatal cases. Am. J. Gastroenterol. 100, 169-176.

Sportelli, M. C., Izzi, M., Kukushkina, E. A., Hossain, S. I., Picca, R. A., Ditaranto, N., et al. (2020). Can nanotechnology and materials science help the fight against SARS-CoV-2? Nanomaterials 10:802. doi: 10.3390/nano1004 0802

Sun, P., Qie, S., Liu, Z., Ren, J., Li, K., and Xi, J. (2020). Clinical characteristics of hospitalized patients with SARS-CoV-2 infection: a single arm meta-analysis. J. Med. Virol. 92, 612-617. doi: 10.1002/jmv.25735

The Lancet Infectious Diseases (2020). Challenges of coronavirus disease 2019. Lancet Infect. Dis. 20:261. doi: 10.1016/s1473-3099(20)30072-4

Tong, Z. D., Tang, A., Li, K. F., Li, P., Wang, H. L., Yi, J. P., et al. (2020). Potential presymptomatic transmission of SARS-CoV-2, Zhejiang Province, China, 2020. Emerg. Infect. Dis. 26, 1052-1054. doi: 10.3201/eid2605.200198

van Doremalen, N., Bushmaker, T., Karesh, W. B., and Munster, V. J. (2014). Stability of middle east respiratory syndrome coronavirus in milk. Emerg. Infect. Dis. 20, 1263-1264. doi: 10.3201/eid2007.140500

van Doremalen, N., Bushmaker, T., Morris, D. H., Holbrook, M. G., Gamble, A., Williamson, B. N., et al. (2020). Aerosol and surface stability of SARS-CoV-2 as compared with SARS-CoV-1. N. Engl. J. Med. 382, 1564-1567.

Walther, B. A., and Ewald, P. W. (2004). Pathogen survival in the external environment and the evolution of virulence. Biol. Rev. 79, 849-869. doi: 10 . $1017 /$ s1464793104006475

Wang, Y., Wang, Y., Chen, Y., and Qin, Q. (2020). Unique epidemiological and clinical features of the emerging 2019 novel coronavirus pneumonia (COVID19) implicate special control measures. J. Med. Virol. 92, 568-576. doi: 10.1002/ jmv. 25748

Warnes, S. L., Little, Z. R., and Keevil, C. W. (2015). Human coronavirus 229E remains infectious on common touch surface materials. mBio 6:e1697-15.

Wei, W. E., Li, Z., Chiew, C. J., Yong, S. E., Toh, M. P., and Lee, V. J. (2020). Presymptomatic transmission of SARS-CoV-2 - Singapore, January 
23-March 16, 2020. Morb. Mortal. Wkly. Rep. 69, 411-415. doi: 10.15585/ mmwr.mm6914e1

World Health Organization [WHO] (2020). Coronavirus Disease (COVID-19) Pandemic. Geneva: WHO.

Ye, F., Xu, S., Rong, Z., Xu, R., Liu, X., Deng, P., et al. (2020). Delivery of infection from asymptomatic carriers of COVID-19 in a familial cluster. Int. J. Infect. Dis. 94, 133-138. doi: 10.1016/j.ijid.2020. 03.042

Yépiz-Gómez, M. S., Gerba, C. P., and Bright, K. R. (2013). Survival of respiratory viruses on fresh produce. Food Environ. Virol. 5, 150-156. doi: 10.1007/s12560013-9114-4

Yu, P., Zhu, J., Zhang, Z., Han, Y., and Huang, L. (2020). A familial cluster of infection associated with the 2019 novel coronavirus indicating potential person-to-person transmission during the incubation period. J. Infect. Dis. 221, 1757-1761. doi: 10.1093/infdis/jiaa077
Zhang, H., Chen, R., Chen, J., and Chen, B. (2020). COVID-19 Transmission within a family cluster in Yancheng, China. Front. Med. 7:387. doi: 10.3389/ fmed.2020.00387

Conflict of Interest: The authors declare that the research was conducted in the absence of any commercial or financial relationships that could be construed as a potential conflict of interest.

Copyright (c) 2020 Olaimat, Shahbaz, Fatima, Munir and Holley. This is an openaccess article distributed under the terms of the Creative Commons Attribution License (CC BY). The use, distribution or reproduction in other forums is permitted, provided the original author(s) and the copyright owner(s) are credited and that the original publication in this journal is cited, in accordance with accepted academic practice. No use, distribution or reproduction is permitted which does not comply with these terms. 